\title{
BRIEF COMMUNICATION OPEN AgBR1 antibodies delay lethal Aedes aegypti-borne West Nile virus infection in mice
}

\author{
Ryuta Uraki (iD), Andrew K. Hastings ${ }^{1}$, Doug E. Brackney ${ }^{2}$, Philip M. Armstrong ${ }^{2}$ and Erol Fikrig (iD) ${ }^{1,3}$
}

West Nile virus (WNV) is transmitted by mosquitoes and can cause severe disease, including meningoencephalitis. AgBR1 is a mosquito salivary protein that enhances Aedes aegypti mosquito-borne Zika virus pathogenesis in mice. Here, we show that AgBR1 antibodies reduce the initial West Nile viral load and delay lethal infection after feeding by an infected Aedes aegypti mosquito. Targeting AgBR1 may therefore be incorporated into strategies to prevent mosquito-transmitted West Nile virus infection.

npj Vaccines (2019)4:23; https://doi.org/10.1038/s41541-019-0120-x

\section{INTRODUCTION}

West Nile virus (WNV) is a single-stranded positive-sense RNA virus in the genus Flavivirus, which normally circulates in a birdmosquito transmission cycle and is a common human mosquitoborne flaviviral infection in North America and other regions in the world. ${ }^{1-3}$ WNV can also infect horses, and other non-avian vertebrate hosts. ${ }^{4}$ Despite substantial efforts, effective FDAapproved preventive or therapeutic measures are not yet available. ${ }^{1,4,5}$

Culex mosquito spp., now endemic in tropical and subtropical regions as well as more temperate areas, are the major vectors for WNV worldwide. ${ }^{5}$ However, the virus also has been isolated from Aedes aegypti mosquitoes, which is present in tropical and subtropical locations as well ${ }^{6,7}$ and are a potential threat for transmission of WNV to humans. ${ }^{8}$ Although the vector competence of WNV in Ae. aegypti is lower than that of Culex spp., multiple factors can affect vector distribution, including climate change, and this may influence the vectorial capacity of Aedes mosquitoes for WNV in the future., 90 Moreover, in laboratory studies, Ae. aegypti readily feed on mice and a well-annotated whole genome sequence is available. ${ }^{11}$

When mosquitoes take a blood meal, they inoculate saliva into the skin. ${ }^{12}$ Mosquito saliva contains molecules which modulate various host responses, including coagulation, platelet aggregation, thrombin activation, vasodilation, and other mammalian host pathways. ${ }^{13,14}$ Previous studies, including some from our group, demonstrate that components of saliva enhance the pathogenicity and transmission of arboviruses including WNV, dengue, Zika, and Semliki Forest viruses, suggesting that certain salivary proteins are important for influencing flavivirus infectivity during transmission from vector to host. ${ }^{15-19}$ Recently, we identified Ae. aegypti AgBR1 as an antigenic protein in vertebrate hosts fed upon by Ae. aegypti mosquitoes. ${ }^{20}$ The expression of AgBR1 in the salivary glands is up-regulated after blood feeding and AgBR1 belongs to a family of proteins that have lost chitinolytic activity, ${ }^{21,22}$ however, the function of this protein in the vertebrate host remains unclear. We showed that Ae. aegypti AgBR1 modulates the early immune response in the murine skin following mosquito bite and that immunization against AgBR1 partially protects mice from a lethal mosquito-borne Zika virus infection. ${ }^{20}$ To determine whether this effect extends beyond Zika virus to another flavivirus, we examined the influence of AgBR1 antibodies against Ae. aegypti-borne WNV infection in mice.

\section{RESULTS AND DISCUSSION}

To determine whether targeting AgBR1 altered pathogenesis during mosquito-borne WNV infection, we passively immunized mice with AgBR1 antiserum before challenging them with WNV by mosquito bite. Ae. aegypti mosquitoes were used as a vector model, since the well-annotated whole genome sequence and easy maintenance make this species ideal for laboratory viral transmission studies. ${ }^{11,23}$ Wild type Swiss Webster mice were administered AgBR1 or control antiserum and $24 \mathrm{~h}$ later were bitten by WNV-infected Ae. aegypti mosquitoes (Fig. 1a). Passive immunization with AgBR1 antiserum significantly reduced WNV RNA levels in the murine bloodstream at an early stage (3 days) of infection (Fig. 1b). Our group and others have previously demonstrated that components of mosquito saliva modulate local host responses and recruit several immune cells which can be target of virus replication. ${ }^{17,20,24}$ This may lead to virus dissemination at an earlier, rather than later, time point. Although we were not able to detect a significant difference at day 1 , it may be due to the complex interplay of recruited immune cells at the bite site, which leads to shifting populations of WNV-susceptible cells over the first hours and days of infection. In addition, pretreatment with AgBR1 antiserum delayed virally-induced weight loss (Fig. 1c) and prolonged median survival time of mice by $20 \%$ (Fig. 1d). As the mosquito-borne WNV infection model used in this study (survival rate: $0 \%$, median survival time: 7 days in control) is much more virulent than our previous mosquitoborne Zika infection model (survival rate: $30-45 \%$, mean survival time: $12-25$ days in control) ${ }^{20}$ the 1.5 -day delay of fatal outcome is noteworthy. Overall, our results indicate that blocking

\footnotetext{
${ }^{1}$ The Anlyan Center for Medical Research and Education, Department of Internal Medicine, Section of Infectious Diseases, Yale University School of Medicine, 300 Cedar Street,

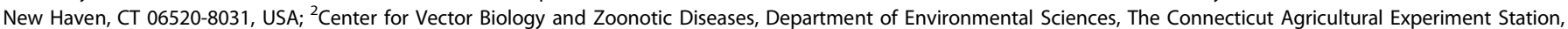
New Haven 06511 CT, USA and ${ }^{3}$ Howard Hughes Medical Institute, Chevy Chase, MD 20815, USA

Correspondence: Erol Fikrig (erol.fikrig@yale.edu)
}

Received: 26 March 2019 Accepted: 10 June 2019

Published online: 08 July 2019 
(a)

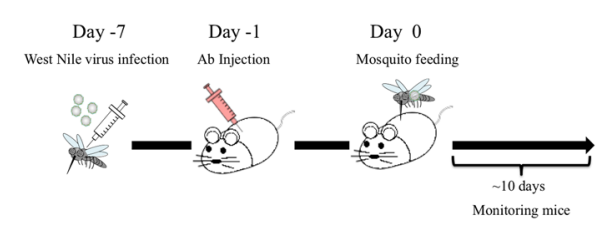

(b)

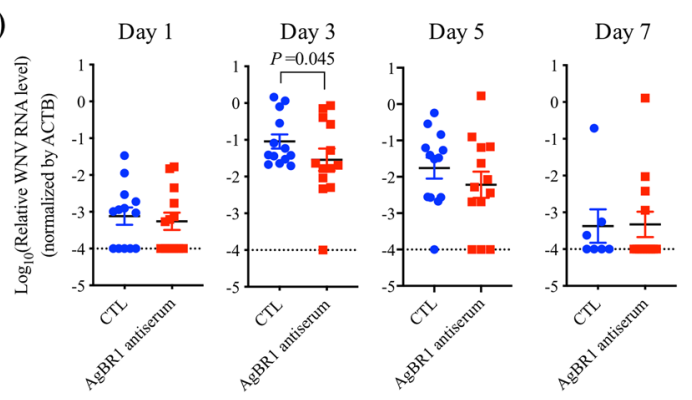

(c)

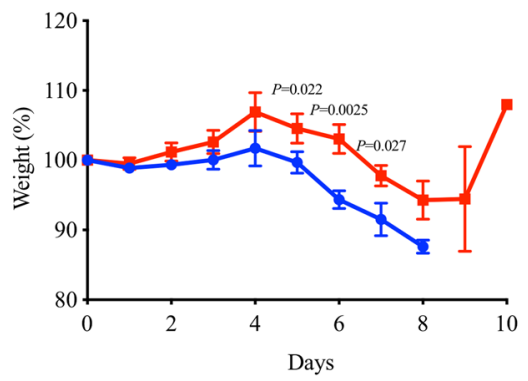

(d)

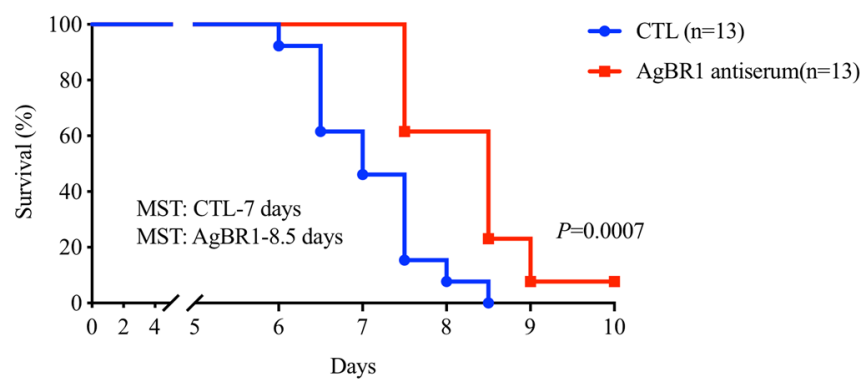

Fig. 1 AgBR1 antiserum protects mice from mosquito-borne WNV virus infection. a The schematic of the experiment. Mice were administrated AgBR1 antiserum one day before WNV-infected mosquito feeding. Immunized mice were monitored for survival for 10 days after infected mosquito feeding. $\mathbf{b}$ The virus levels in blood of mice fed by an infected mosquito. Blood was collected every other day for 7 days from mice fed on by WNV-infected mosquitoes and analyzed by qRT-PCR. WNV RNA levels were normalized to mouse $\beta$ actin RNA levels. Mice immunized with naïve serum served as controls. Error bars represent mean \pm SEM. Each data point represents one mouse. Normalized viral RNA levels were analyzed using one-tailed Wilcoxon-Mann-Whitney test $(n=13 /$ each group biologically independent samples pooled from three separate experiments). $\mathrm{c}$ The weight of mice fed by an infected mosquito. Mice were monitored daily after WNV infection. Error bars represent mean \pm SEM. Weight at each time point were compared using one-tailed Wilcoxon-Mann-Whitney test $(n=13 /$ each group biologically independent samples pooled from three separate experiments). $\mathbf{d}$ Survival was assessed by a Gehan-Wilcoxon test ( $n=13$ /each group biologically independent samples pooled from three separate experiments)

AgBR1 suppresses virus replication and/or dissemination at early time points and alters mosquito-borne WNV infection.

We then determined whether the effects observed with AgBR1 were specific to this protein or if any antigenic Ae. aegypti salivary gland protein was capable of affecting WNV infection. We chose an additional protein, the putative $34 \mathrm{kDa}$ family secreted salivary protein (SP), which was the top hit from our previous yeast surface display screen. Sera from mice bitten by mosquitoes showed strong reactivity to the SP protein. ${ }^{20}$ SP has also been reported as a salivary gland protein that was upregulated during flavivirus infection. ${ }^{18}$ We performed identical passive immunization experiments using SP antiserum. SP antiserum did not alter viremia, weight loss or survival time after lethal mosquito-borne WNV infection (Supplementary Fig. 1).

Our previous study showed that AgBR1 antiserum suppresses early host responses at bitten sites after Zika virus-infected mosquito feedings in AG129 mice. ${ }^{20}$ Therefore, we examined whether AgBR1 antibodies alter the early host responses after feeding by mosquitoes infected with WNV. Proinflammatory genes including $\| 1 b$, $I 6$, and Tnfa, were significantly suppressed at the bite site in mice treated with AgBR1 antiserum at $6 \mathrm{~h}$ post feeding (Fig. 2a). In addition to these genes, we examined the expression levels of $M m p 9$, which is previously reported to play an important role in WNV entry into the brain, ${ }^{25}$ and those of Nlrp3, which is a key molecule of NLRP3 inflammasome that drives IL-1 $\beta$ signaling and is involved in WNV control in the central nervous system (CNS). ${ }^{26}$ As shown in Fig. 2a, Mmp9 and Nlrp3 genes were significantly suppressed in AgBR1 antiserum-treated mice at $6 \mathrm{~h}$ post feedings. Interestingly, we did not see differences in the expression level of any of these genes $24 \mathrm{~h}$ after bites with WNVinfected mosquitoes (Fig. 2b). In our previous study of AgBR1 and Zika virus, we observed significant suppression of several proinflammatory genes and cytokines at $24 \mathrm{~h}$ post Zika virusinfected mosquitoes, and there are a few underlying differences in the experimental design that could explain these differences. One major difference is that the current study of WNV took advantage of immunocompetent wild type mice, as contrasted with the immuno-incompetent Ifnar1 ${ }^{-\prime-}$ Ifngr $^{-/-}$mice used for the Zika virus study. Therefore, it is possible that the difference of mouse models could cause the time lag of early host responses. Overall, these results demonstrated that AgBR1 antiserum suppresses the early local host responses after WNV-infected mosquito feedings, suggesting that the suppression of host responses by AgBR1 antibodies leads to the delay of viral dissemination and fatal outcome.

AgBR1 antiserum also specifically recognizes a protein in Culex pipiens salivary glands (Suplementary Fig. 2). Since Ae. aegypti AgBR1 has high homology (amino acid identities $=80 \%$ ) with Culex spp. chitotriosidase-1 protein, which is predicted in silico to be secreted from the salivary gland, we hypothesize that this Culex protein recognized by AgBR1 antiserum may have similar function during WNV infection. Further work will be needed to examine whether immunization against the Culex homologous protein has a protective effect in WNV infection transmitted by Culex mosquitoes.

In conclusion, our study demonstrated that passive immunization with AgBR1 antiserum delays lethal Ae. aegypti-borne WNV infection in mice, as we have previously shown for Zika virus infection transmitted by the same mosquitoes. Therefore, given the results of this and other studies, we suggest that the strategy of targeting individual arthropod salivary factors such as AgBR1 might be broadly applicable to other mosquito-borne pathogens. 
(a) 6 hours post infection

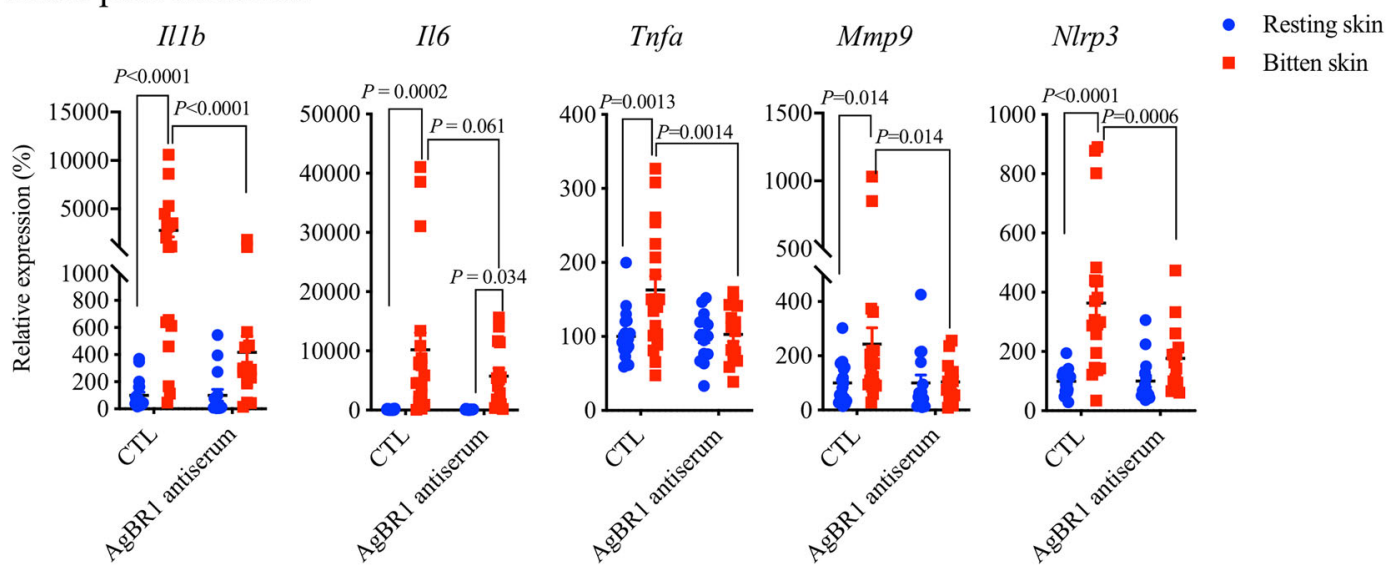

(b) 24 hours post infection
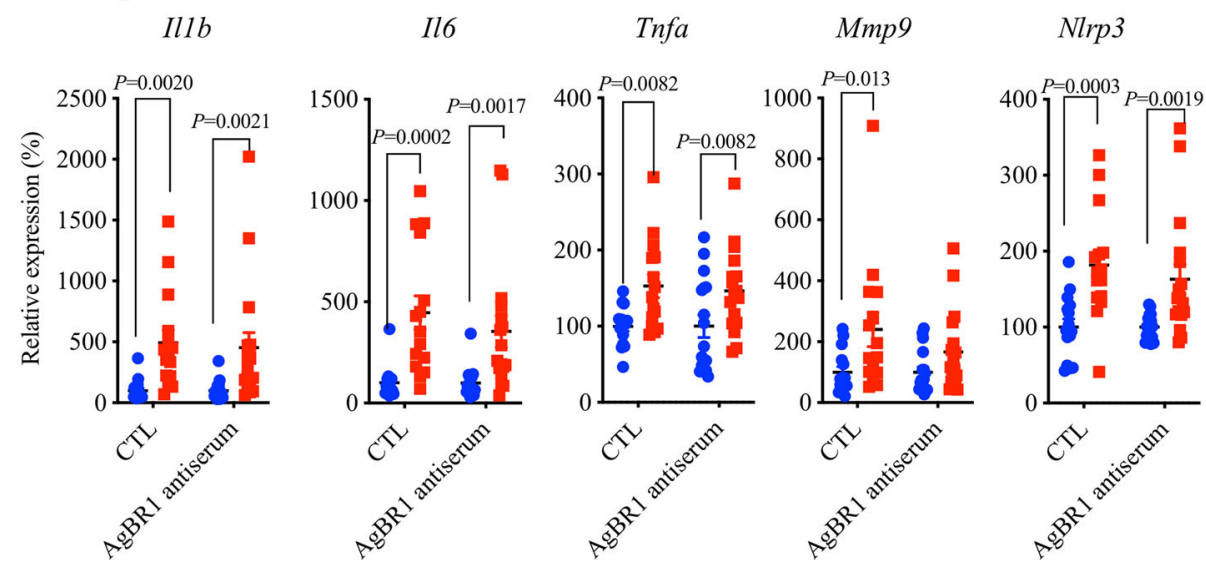

Fig. 2 AgBR1 antiserum modulates host responses at the WNV-infected mosquito bite site. The expression levels of several cytokines were analyzed by qRT-PCR at $\mathbf{a} 6 \mathrm{~h}$ or $\mathbf{b} 24 \mathrm{~h}$ after bites of infected mosquitoes, which is normalized to mouse $\beta$ actin RNA levels. Error bars represent mean \pm SEM. Each dot represents one bitten or control site. Significance was determined by two-way ANOVA test ( $6 \mathrm{~h}$; $n=19 /$ control group, $n=15 / \mathrm{AgBR} 1$ antiserum group, $24 \mathrm{~h} ; n=15 /$ control group, $n=17 / \mathrm{AgBR} 1$ antiserum group biologically independent samples pooled from two separate experiments)

\section{METHODS}

Ethics statement

All experiments were performed in accordance with guidelines from the Guide for the Care and Use of Laboratory Animals of the NIH. The animal experimental protocols were approved by the Institutional Animal Care and Use Committee (IACUC) at the Yale University School of Medicine. Procedures for handling and care of animals were approved by and performed under the Animal Care and Use Committee at The Connecticut Agricultural Experiment Station. All infection experiments were performed in a biosafety level 3 animal facility, according to Yale University and the Connecticut Agricultural Experiment Station regulations. Every effort was made to minimize murine pain and distress. Mice were anesthetized with ketamine/xylazine for mosquito infection experiments and euthanized as suggested by the IACUC.

\section{Mosquitoes and animals}

Ae. aegypti (Orlando strain, collected from Orlando, $\mathrm{FL}$ in 1952) and $C X$. pipiens mosquitoes were maintained on $10 \%$ sucrose feeders inside a $12^{\prime \prime} \times 12^{\prime \prime} \times 12^{\prime \prime}$ metal mesh cage (BioQuip \#1450B) at $28^{\circ} \mathrm{C}$ and $\sim 80 \%$ humidity. Eggs were generated via blood meal feeding on an artificial membrane feeder with defibrinated sheep's blood (Hemostat Laboratories). All mosquitoes were housed in a warm chamber in a space approved for BSL3 and ACL3 research. Four-week-old male mice (Swiss Webster mice from Charles River) were used in the WNV infection studies. All mice were kept in a pathogen-free facility at the Connecticut Agricultural Experiment Station.

\section{Mosquito injection and dissections}

For WNV injection, WNV-filled needles were carefully inserted into the thorax of each mosquito and $69 \mathrm{nl}$ of virus $\left(3.4 \times 10^{3} \mathrm{PFU}\right)$ was injected using a Nanoject II auto-nanoliter injector (Drummond). Infected mosquitoes were placed back in paper cups with mesh lids and maintained in triple containment for 7 days in a warm chamber. After feeding infected mosquitoes on naïve mice, they were knocked-down on ice and salivary glands were dissected to examine the virus levels.

\section{Passive immunization studies}

Mice were injected intraperitoneally with $150 \mu$ per animal of AgBR1 or SP antiserum or naive rabbit serum one day before challenge. On the following day, mice were anesthetized with ketamine-xylazine and fed on by a single WNV-infected mosquito per mouse. The blood of fed mice was collected at 1, 3, 5, and 7 days post infection. Survivals and weights were monitored every day. Mice exhibiting weight loss of $>20 \%$ of initial body weight or neurologic disease were euthanized. Viremia levels were examined at 1,3,5, and 7 days post infection by quantitative real time-PCR.

Analysis of local immune responses after bites of West Nile virusinfected mosquitoes

Mice were passively immunized with either AgBR1 or naive antiserum $24 \mathrm{~h}$ prior to allowing infected Ae. aegypti mosquitoes to feed on the left ear. After 6 or $24 \mathrm{~h}$ post feedings, mice were euthanized, and the locations bitten by mosquitoes and naïve locations on the opposite ear were punched using a Disposable Biopsy Punch. Total RNA was extracted by the 
RNeasy Fibrous Tissue Mini Kit (QIAGEN) according to the manufacturer's instructions. For quantitative RT-PCR, the CDNA was generated with iScript cDNA Synthesis Kit (Bio-rad) according to manufacturer's protocol. Gene expression was examined by qRT-PCR using IQ ${ }^{\mathrm{TM}}$ SYBR Green Supermix. Target gene mRNA levels were normalized to mouse $\beta$ actin RNA levels according to the $2^{-\Delta \Delta C t}$ calculations. The qRT-PCR primer sequences are available upon request.

\section{Immunoblot}

Three sets of salivary glands from $A$ e. aegypti and $C x$. pipiens were placed in $20 \mu \mathrm{l}$ Novex 2x Tris-Glycine SDS Sample Buffer, heated to $85^{\circ} \mathrm{C}$ for $5 \mathrm{~min}$, diluted 1:1 with water and the whole sample was loaded on a $16 \%$ Trisglycine gel. AgBR1 and homologous proteins were examined with AgBR1 antiserum (1:1000 dilution), followed by incubation with HRP-conjugated secondary antibodies.

\section{Statistical analysis}

GraphPad Prism software was used to analyze all the data. Mouse $\beta$ actinnormalized viral RNA levels and body weights were analyzed using the Wilcoxon-Mann-Whitney test. Host responses in vivo was performed using a two-way ANOVA for multiple comparisons. Survival was assessed by a Gehan-Wilcoxon test. A $p$ value of $<0.05$ was considered statistically significant.

\section{Reporting summary}

Further information on experimental design is available in the Nature Research Reporting Summary linked to this article.

\section{DATA AVAILABILITY}

The data that support the findings of this study are available from the corresponding authors upon request.

\section{ACKNOWLEDGEMENTS}

This work was supported by NIH grants Al145779, Al127865, Al08992 and the Japan Society for the Promotion of Science Overseas Research Fellowships. E.F. is an Investigator with the Howard Hughes Medical Institute.

\section{AUTHOR CONTRIBUTIONS}

R.U., A.K.H. and E.F. designed the experiments; R.U., A.K.H., D.E.B. and P.M.A. performed the experiments and analyzed the data; R.U. and E.F. wrote the paper. All authors reviewed, critiqued, and provided comments on the text.

\section{ADDITIONAL INFORMATION}

Supplementary information accompanies the paper on the npj Vaccines website (https://doi.org/10.1038/s41541-019-0120-x).

Competing interests: The authors declare no competing interests.

Publisher's note: Springer Nature remains neutral with regard to jurisdictional claims in published maps and institutional affiliations.

\section{REFERENCES}

1. Kramer, L. D., Styer, L. M. \& Ebel, G. D. A global perspective on the epidemiology of West Nile virus. Annu. Rev. Entomol. 53, 61-81 (2008).

2. McGee, C. E., Schneider, B. S., Girard, Y. A., Vanlandingham, D. L. \& Higgs, S. Nonviremic transmission of West Nile virus: evaluation of the effects of space, time, and mosquito species. Am. J. Trop. Med. Hyg. 76, 424-430 (2007).

3. Reisen, W. \& Brault, A. C. West Nile virus in North America: perspectives on epidemiology and intervention. Pest Manag Sci. 63, 641-646 (2007).
4. Nikolay, B. A review of West Nile and Usutu virus co-circulation in Europe: how much do transmission cycles overlap? Trans. R. Soc. Trop. Med. Hyg. 109, 609-618 (2015).

5. Petersen, L. R., Brault, A. C. \& Nasci, R. S. West Nile virus: review of the literature. JAMA 310, 308-315 (2013).

6. Vogels, C. B., Goertz, G. P., Pijlman, G. P. \& Koenraadt, C. J. Vector competence of European mosquitoes for West Nile virus. Emerg. Microbes Infect. 6, e96 (2017).

7. Gould, E. A. \& Solomon, T. Pathogenic flaviviruses. Lancet 371, 500-509 (2008).

8. Vanlandingham, D. L. et al. Relative susceptibilties of South Texas mosquitoes to infection with West Nile virus. Am. J. Trop. Med. Hyg. 77, 925-928 (2007).

9. Andersen, L. K. \& Davis, M. D. Climate change and the epidemiology of selected tick-borne and mosquito-borne diseases: update from the International Society of Dermatology Climate Change Task Force. Int J. Dermatol 56, 252-259 (2017).

10. Turell, M. J., O'Guinn, M. L., Dohm, D. J. \& Jones, J. W. Vector competence of North American mosquitoes (Diptera: Culicidae) for West Nile virus. J. Med. Entomol. 38, 130-134 (2001).

11. Matthews, B. J. et al. Improved reference genome of Aedes aegypti informs arbovirus vector control. Nature 563, 501-507 (2018).

12. Coutinho-Abreu, I. V., Guimaraes-Costa, A. B. \& Valenzuela, J. G. Impact of insect salivary proteins in blood feeding, host immunity, disease, and in the development of biomarkers for vector exposure. Curr. Opin. Insect Sci. 10, 98-103 (2015).

13. Fontaine, A. et al. Implication of haematophagous arthropod salivary proteins in host-vector interactions. Parasit. Vectors 4, 187 (2011).

14. Hayashi, H. et al. Anopheline anti-platelet protein from a malaria vector mosquito has anti-thrombotic effects in vivo without compromising hemostasis. Thromb. Res. 129, 169-175 (2012).

15. Styer, L. M. et al. Mosquito saliva causes enhancement of West Nile virus infection in mice. J. Virol. 85, 1517-1527 (2011).

16. Conway, M. J. et al. Mosquito saliva serine protease enhances dissemination of dengue virus into the mammalian host. J. Virol. 88, 164-175 (2014).

17. Pingen, M. et al. Host Inflammatory Response to Mosquito Bites Enhances the Severity of Arbovirus Infection. Immunity 44, 1455-1469 (2016).

18. Conway, M. J. et al. Aedes aegypti D7 Saliva Protein Inhibits Dengue Virus Infection. PLoS Negl. Trop. Dis. 10, e0004941 (2016).

19. Jin, L. et al. Salivary factor LTRIN from Aedes aegypti facilitates the transmission of Zika virus by interfering with the lymphotoxin-beta receptor. Nat. Immunol. 19, 342-353 (2018).

20. Uraki, R. et al. Aedes aegypti AgBR1 antibodies modulate early Zika virus infection of mice. Nat. Microbiol., https://doi.org/10.1038/s41564-019-0385-x (2019).

21. Shi, L. \& Paskewitz, S. M. Identification and molecular characterization of two immune-responsive chitinase-like proteins from Anopheles gambiae. Insect Mol. Biol. 13, 387-398 (2004).

22. Ribeiro, J. M. et al. An annotated catalogue of salivary gland transcripts in the adult female mosquito, Aedes aegypti. BMC Genom. 8, 6 (2007).

23. Cheng, G. et al. A C-type lectin collaborates with a CD45 phosphatase homolog to facilitate West Nile virus infection of mosquitoes. Cell 142, 714-725 (2010).

24. Hastings, A. K. et al. Aedes aegypti NeSt1 protein enhances Zika virus pathogenesis by activating neutrophils. J. Virol., https://doi.org/10.1128/JVI.00395-19 (2019).

25. Wang, P. et al. Matrix metalloproteinase 9 facilitates West Nile virus entry into the brain. J. Virol. 82, 8978-8985 (2008).

26. Ramos, H. J. et al. IL-1beta signaling promotes CNS-intrinsic immune control of West Nile virus infection. PLoS Pathog. 8, e1003039 (2012).

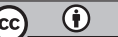

Open Access This article is licensed under a Creative Commons Attribution 4.0 International License, which permits use, sharing, adaptation, distribution and reproduction in any medium or format, as long as you give appropriate credit to the original author(s) and the source, provide a link to the Creative Commons license, and indicate if changes were made. The images or other third party material in this article are included in the article's Creative Commons license, unless indicated otherwise in a credit line to the material. If material is not included in the article's Creative Commons license and your intended use is not permitted by statutory regulation or exceeds the permitted use, you will need to obtain permission directly from the copyright holder. To view a copy of this license, visit http://creativecommons. org/licenses/by/4.0/.

(c) The Author(s) 2019 